\title{
Glans penis augmentation using hyaluronic acid for the treatment of premature ejaculation: a narrative review
}

\author{
Fares Kosseifi ${ }^{1,2}$, Ala Chebbi ${ }^{2}$, Nehme Raad $^{1}$, Antoinette Ndayra ${ }^{3}$, Raed El Samad ${ }^{4}$, Kamal Achkar ${ }^{4}$, \\ Xavier Durand ${ }^{2}$, Antoine Noujeim ${ }^{1}$ \\ ${ }^{1}$ Division of Urology, Department of Surgery, Faculty of Medical Sciences, Lebanese University, Beirut, Lebanon; ${ }^{2}$ Department of Urology, Saint- \\ Joseph Hospital, Paris, France; ${ }^{3}$ Department of Psychology, Faculty of Arts and Sciences, University of Balamand, Beirut, Lebanon; ${ }^{4}$ Department of \\ Urology, GHPSO Hospital, Creil, France \\ Contributions: (I) Conception and design: F Kosseifi, A Chebbi, N Raad; (II) Administrative support: K Achkar, X Durand, A Noujeim; (III) Provision \\ of study materials or patients: F Kosseifi, A Chebbi, N Raad, R El Samad; (IV) Collection and assembly of data: F Kosseifi, A Chebbi, A Ndayra; (V) \\ Data analysis and interpretation: All authors; (VI) Manuscript writing: All authors; (VII) Final approval of manuscript: All authors. \\ Correspondence to: Fares Kosseifi. Faculty of Medical Sciences, Lebanese University, Beirut, Lebanon. Email: fareskosseifi@live.com.
}

\begin{abstract}
Premature ejaculation (PE) is the most common self-reported male sexual disorder estimated to occur in approximately $5 \%$ of men in the general community. Penile hypersensitivity is thought to be an etiologic factor of lifelong PE. The role of glans penis augmentation using injectable hyaluronic acid (HA) for the treatment of PE is debatable and remains to be confirmed. The creation of a barrier at the level of the glans, by the bulking agent blocking accessibility and inhibiting the tactile stimuli to reach the dorsal nerve of the penis (branch of the pudendal nerve) receptors, is the theory behind the effectiveness of HA in the field of PE. We reviewed the literature using PubMed and searched for the following keywords: premature ejaculation, glans penis and HA, over the last 20 years. Five studies were found. These studies showed that HA injection could significantly increase IELT (2.43- to 4.46-fold), and this increase could persist for long term (up to 5 years). No serious adverse reactions were reported besides transient discoloration and swelling of the glans that recovered to normal within 2 weeks. Many techniques were discussed, their effectiveness remains to be proved. However, proper patient selection and mastering the esthetics of the technique, by adequate surgical training, is necessary in order to achieve the optimal results.
\end{abstract}

Keywords: Hyaluronic acid (HA); glans penis; premature ejaculation (PE); intravaginal ejaculation latency time (IELT); augmentation

Submitted Jun 21, 2020. Accepted for publication Oct 14, 2020.

doi: $10.21037 /$ tau-20-1026

View this article at: http://dx.doi.org/10.21037/tau-20-1026

\section{Introduction}

Premature ejaculation (PE) is the most common selfreported male sexual disorder. The two most relevant and evidence-based definitions of $\mathrm{PE}$ are those of International Society for Sexual Medicine (ISSM) and American Psychiatric Association (DSM-V) (1). The prevalence of PE is inconsistently variable throughout history and sometimes even overestimated. However, an approximately $5 \%$ prevalence has been reported as most consistent with Clinical PE per Althof et al. in 2014 (2). Both definitions agree on the chronic aspect of this disorder and the relatively short time interval between penetration and ejaculation ( $\leq 1 \mathrm{~min})$, the diminutive or the absence of voluntary control of ejaculation, and the negative consequences, such as distress in nearly all vaginal penetrations $(3,4)$. A third definition has been recently proposed by the American Urological Association Guidelines 2020, PE is defined as poor ejaculatory control, associated bother, and ejaculation within about 2 minutes of initiation of penetrative sex that has been always present for lifelong $\mathrm{PE}$, and ejaculation latency that is markedly reduced from prior sexual experience for acquired PE (5). Two main subtypes of $\mathrm{PE}$ are distinguished based on the duration of 


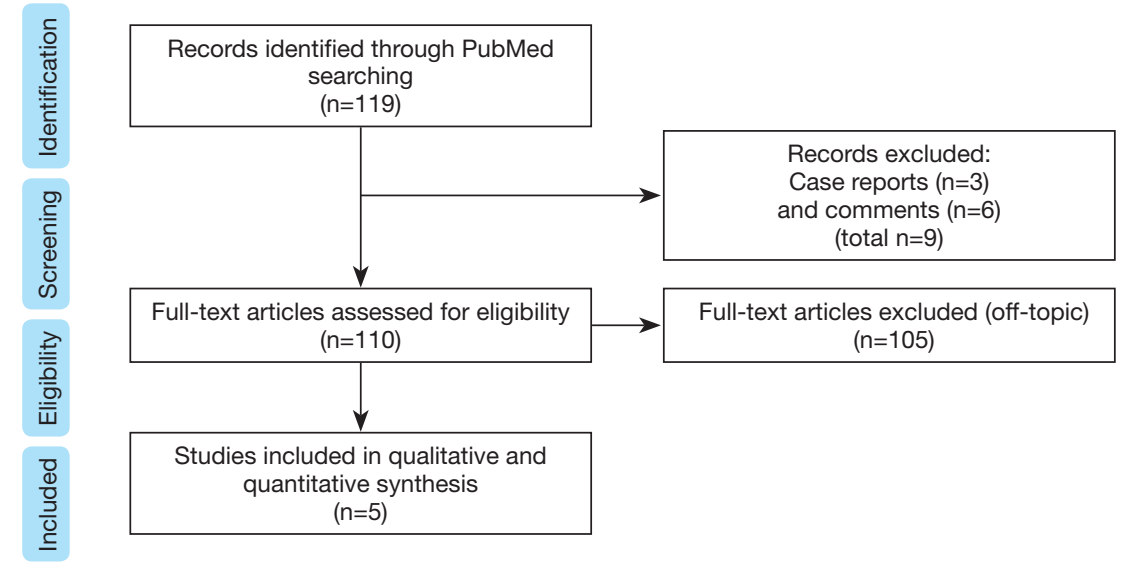

Figure 1 Flowchart.

intravaginal ejaculation latency time (IELT), frequency of reports, and course in life. In lifelong PE, early ejaculation is present at nearly every intercourse within 30 to 60 seconds in the majority of cases (80-90\%) or between 1 to 2 minutes (10-20\%). Conversely, acquired PE occurs at some point in life, it is often situational and preceded by normal ejaculation experiences.

Lifelong PE may be due to 5-HT2C hyposensitivity and/ or 5-HT1A hypersensitivity but also to peripheral penile hypersensitivity $(1,6,7)$.

The sensory innervation of the penis is provided by the deepest divisions of the pudendal nerve which pass through the Alcock canal and continue as the dorsal nerves of the penis. These nerves richly supply the glans. They travel alongside the dorsal arteries. On the other side, small branches of the perineal nerve supply the ventrum of the penis (1).

Several forms of pharmacotherapy have been used in the treatment of PE. These include the use of topical local anesthetics, long- or rapid-acting and short half-life SSRIs (dapoxetine), tramadol, phosphodiesterase type 5 inhibitors, and $\alpha$-adrenergic blockers. Psychosexual cognitive behavioral therapy and coaching has an important role as an adjunct to pharmacotherapy. Surgical neurotomy, percutaneous cryoneurolysis or neuromodulation of the dorsal penile nerve are surgical therapies described in some studies.

Among them, the role of glans penile augmentation remains unclear and is not recommended in the management of PE according to AUA, EAU and ISSM guidelines. It should be considered experimental and more safety studies must be conducted $(3,5,8)$. In addition, ISSM guidelines state that it may be associated with permanent loss of sexual function and is thus currently not recommended.

This review aims to describe the techniques and outcomes of glans penile augmentation in the treatment of $\mathrm{PE}$ and to assess their safety and efficacy.

We present the following article in accordance with the Narrative Review reporting checklist (available at http:// dx.doi.org/10.21037/tau-20-1026).

\section{Methods}

We reviewed the literature using PubMed and searched for the following keywords: premature ejaculation, glans penis and hyaluronic acid (HA) (1) over the last 20 years (1/1/2000 to $1 / 1 / 2020)$ using this combination of Boolean operators "Premature ejaculation AND ((glans penis) OR (hyaluronic acid))". The articles were selected according to their relevance to this review and language (English). The references from all the articles found were searched for further relevant literature. Case reports and comments were excluded (Figure 1). Only 5 studies were found. We thus analyzed these studies (Table 1) and discussed the different surgical techniques and their consequent outcomes.

\section{Results}

\section{Physiological rational}

Penile sensation is the result of multiple interfering factors including the dorsal nerve distributions, the number of receptors, the threshold of these receptors, and the accessibility of the stimuli to these receptors. The creation of a barrier, by the bulking agent blocking accessibility and 


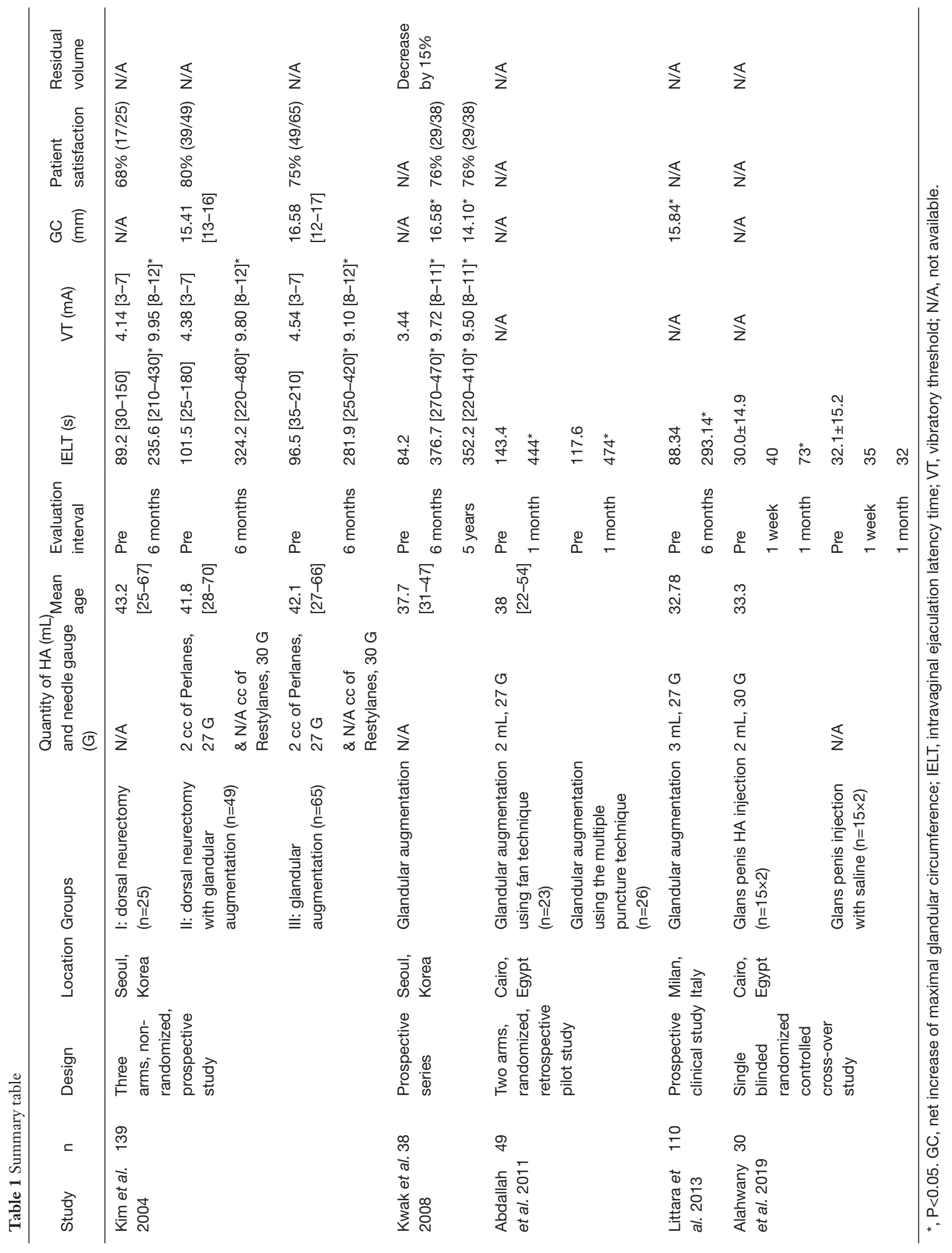



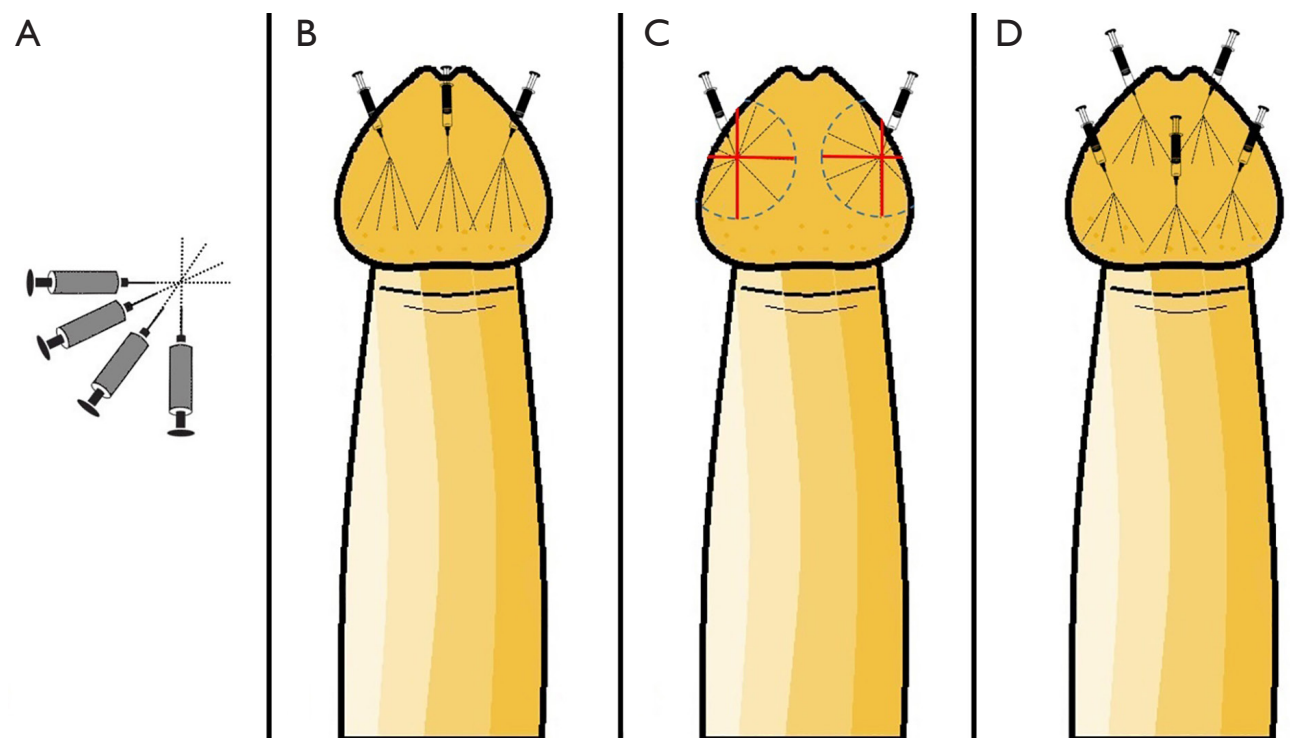

Figure 2 Injection techniques. (A) The fan technique; (B) Kim et al. one-third technique; (C) the three circles technique; (D) the two circular levels technique.

inhibiting the tactile stimuli to reach the nerve receptors, is the theory behind the effectiveness of HA in the field of PE (7). HA can be successfully injected into the dermis of glans penis just above the nerve terminal (9).

HA has been shown to possess many valuable medical properties. It is biocompatible, non-antigenic, nonpyrogenic, non-inflammatory, and nontoxic. It is also easy to use, stable after injection, non-migratory, long lasting but reabsorbable, and natural looking (7). HA has a characteristic of isovolemic degradation (9). The appropriate filler can restore symmetry, volume and create a smooth skin surface (10).

\section{Techniques}

Kim et al. performed glans penis augmentation under local anesthesia, $30 \mathrm{~min}$ after topical application of Emla (lidocaine and prilocaine), 2 cc of injectable HA gel, Perlanes ${ }^{\circledR}$, was injected via 27 -gauge needle. Injection needle was indwelled subcutaneously at proximal onethird from tip of glans to coronal sulcus. Thereafter, HA gel was injected by Fan technique (Figure 2A,B). After injection of Perlanes, undulation of glandular surface was supplemented by injection of Restylanes ${ }^{\circledR}$ via 30 -gauge needle. Both Restylanes and Perlanes are injectable HA gel and have the same composition of $20 \mathrm{mg} / \mathrm{mL}$ of stabilized HA gel. The difference between the products is the size of the gel particles. Approximate number of gel particles is $100,000 / \mathrm{mL}$ in Restylanes and $1,000 / \mathrm{mL}$ in Perlanes. The suppliers recommend 30-gauge needle to inject Restylanes into the mid to upper part of dermis and 27-gauge needle to inject Perlanes into the deep layer of the dermis (7).

Kwak et al. used the same HA gel, Perlanes, but no details concerning the surgical technique were presented (9). However, all the patients included were circumcised.

In the study conducted by Abdallah et al. (11), two techniques were adopted. Group A used Kim et al. 2004 fan technique discussed previously while group B $(n=30)$ received a single injection of $2 \mathrm{~mL}$ of HA gel using the multiple puncture technique. This technique was developed to allow more uniform distribution of the injected material. Using a 27 -gauge needle, multiple points of entry were created starting from proximal onethird of the glans along the coronal sulcus together with the frenulum after application of the topical anesthetic. At each point, only $0.25 \mathrm{~mL}$ was injected.

Littara et al. performed local anesthesia by injecting with a $28-\mathrm{G}$ needle containing $1 \mathrm{~mL}$ of $1 \%$ lidocaine and prilocaine. The circumference of the glans penis was divided into three circles, from the base of the glans at a $1-\mathrm{cm}$ distance from each other. The circles were then divided into quarter circles (Figure 2C). An injection containing $1 \mathrm{~mL}$ HA, Variofill ${ }^{\circledR}$, was performed in the deep dermis into every quarter circle with a $27-\mathrm{G}$ needle for a total of 12 injections 
performed in a single session.

In 2019, Alahwany et al. modified the technique used by Abdallah et al. Under topical anesthesia (30 min of Emla cream), two prefilled $1 \mathrm{ml}$ syringes of cross-linked HA were injected with $30 \mathrm{G}$ needle $\left(\right.$ Teosyal $^{\circledR}$ ) using multiple puncture technique at two circular levels: one at the level of corona and the second one mid-way between the corona and urethral meatus. Six injections were performed at coronal level and four in the second level, each injection containing $0.2 \mathrm{~mL}$ into deep dermis (Figure 2D). All participants were previously circumcised.

\section{Outcomes}

The designs, the results and the conclusions of the different articles are presented within the summary table (Table 1). Vibratory threshold (VT) of glans penis was measured using a biothesiometer.

Interestingly, in all five studies, IELT was significantly increased at every interval evaluation. A rise of 2.92 times at 6 months was demonstrated by Kim et al. with $75 \%$ patient satisfaction. It was increased even more, by 4.46 times at 6 months and 4.18 times at 5 years for Kwak et al. with 76\% overall patient satisfaction. Similarly, an increase of 3.58 times and 2.43 times at 1 month was observed, respectively, for Abdallah et al. and Alahwany et al. Littara et al. also noted an increase by 3.32 times at 6 month.

Kim et al., Kwak et al., Littara et al., and Alahwany et al., all reported no serious adverse reactions in all cases. There was no abnormal reaction in area feeling, texture and color. In most cases, initial discoloration by glandular swelling recovered to normal within 2 weeks. In most patients, local application of anesthetic cream was sufficient $(7,9,10,12)$.

The multiple puncture technique was associated with less pain, because the size of the bulla created is smaller than that created using the fan technique. However, disadvantages include longer injection time and higher risk of bruising as an adverse event in $28.54 \%$ of the cases, which was found in seven cases (out of 49) in the Abdallah et al. study and resolved completely within few days (11). Littara et al. reported no pain under local anesthesia and no inflammatory signs or other adverse reactions were observed in all cases (10).

\section{Discussion}

The efficacy of glans penis augmentation by HA injection was evaluated by IELT, VT, the patient's satisfaction and the partner's satisfaction. The currently available studies showed that HA injection could significantly increase IELT (2.43to 4.46 -fold), and this increase could persist for long term (up to 5 years). This outcome can be explained by reduced sensation threshold of the glans penis. In fact, this result seems promising when compared with that of Dapoxetine 30 or $60 \mathrm{mg}$ taken 1 to 2 hours before intercourse which results in 2.5- and 3.0-fold increase in IELT, increased ejaculatory control, decreased distress, and increased satisfaction (1). The significant improvement observed in the median IELT after glans penis HA injection, remains to be objectively proved with further studies using unified evaluation scores and criteria.

In addition, $\mathrm{HA}$ injection has the advantage over other medical treatment of being injected once every 9-12 months, the absence of systemic side-effects and the absence of negative consequences on sexual desire or fertility. It also has an additional benefit in $\mathrm{PE}$ provided by the positive impact of the enlarged glans on self-esteem and self-confidence (3). Adverse reactions were minimal and merely self-limiting (ecchymosis and injection-site discomfort). HA injections for treatment of PE did not affect other domains of sexual functions. Allergic reactions were not reported $(7,12)$.

Although feasible, the relatively decreased adoption of glans penis augmentation is probably related to its yet unproven outcomes but also to its technically challenging aspects. The first challenge of this procedure is the absence of a precise measure for the needed volume of HA to inject in order to achieve satisfactory, effective but nonexaggerated results. The second challenge is the difficult injection technique. First, in terms of even distribution of the gel through whole glans penis and inevitable minor surface undulation, which originate from the undulation of underlying rete ridge. However, although this undulation sometimes looks unnatural, it does often disappear during glans erection and as a result most patients are still satisfied (8). The second concern is the depth of injection. Too superficial injections can exaggerate these surface undulations and might even cause pressure necrosis due to the lack of elasticity of the glandular skin, while deep injections may not lead to an adequate size augmentation or effective sensory inhibition, and most importantly can lead to deep vascular compromise. Another challenge is the choice of the size and the consistency of the HA gel particles. Medium molecular weight and medium viscosity HA are preferable in terms of ease to inject and esthetic appearance. Large molecular weight and viscous HA are logically less esthetically and practically acceptable 
considering the small surface and space as well as the low elasticity present within the glandular area. Finally, the number and the distribution of injection sites remains to be tailored according to each glans anatomical appearance and surgeons' experience while considering the risk/ benefit ratio. The main risk of multiple injections being the increased incidence of injections sites complications (ecchymosis, pain, local infection...) while the main benefit being esthetically more adequate and homogenous repartition of the filler material throughout the glandular surface.

The reason behind performing a prior circumcision in the setting of glans penis augmentation is probably purely esthetic. However, some studies had shown that distal circumcision itself may be an effective surgical treatment of lifelong $\mathrm{PE}$ in patients with an excessive prepuce $(13,14)$ while others rejected this theory (15).

It is noteworthy to mention that most of these studies (except that of Kwak et al.) lack long term follow-up (Table 1).

Another limitation is patient satisfaction assessment. In fact, two studies only reported the patient satisfaction rate and this was done using a non-validated tool (Table 1).

Finally, it is important to note, in these studies, the small samples size, the extremely variable and different patient related inclusion criteria (Primary PE, secondary PE...) and exclusion criteria (circumcision, previous medical treatment for PE, chronic psychiatric or systemic diseases, alcohol or substance abuse...), as well as the post-operative evaluation criteria (intervals, patient satisfaction, partner satisfaction...). However, proper patient selection is crucial. In fact, the use of glans penis augmentation seems more logical in patients when pharmacotherapy has failed or if there was a good response to topical treatment (16).

\section{Conclusions}

The currently available studies showed the efficacy and safety of HA in the management of PE. However, in order to confirm these results, large randomized prospective studies are required. In addition, to increase the efficacy of glans penis augmentation by filler for $\mathrm{PE}$, proper patient selection is very important, and mastering the esthetics of the technique, by adequate surgical training, is necessary in order to achieve the optimal harmonic appearance.

\section{Acknowledgments}

Funding: None.

\section{Footnote}

Reporting Checklist: The authors have completed the Narrative Review reporting checklist. Available at http:// dx.doi.org/10.21037/tau-20-1026

Peer Review File: Available at http://dx.doi.org/10.21037/ tau-20-1026

Conflicts of Interest: All authors have completed the ICMJE uniform disclosure form (available at http://dx.doi. org/10.21037/tau-20-1026). The authors have no conflicts of interest to declare.

Ethical Statement: The authors are accountable for all aspects of the work in ensuring that questions related to the accuracy or integrity of any part of the work are appropriately investigated and resolved.

Open Access Statement: This is an Open Access article distributed in accordance with the Creative Commons Attribution-NonCommercial-NoDerivs 4.0 International License (CC BY-NC-ND 4.0), which permits the noncommercial replication and distribution of the article with the strict proviso that no changes or edits are made and the original work is properly cited (including links to both the formal publication through the relevant DOI and the license). See: https://creativecommons.org/licenses/by-nc-nd/4.0/.

\section{References}

1. Wein A, Kavoussi L, Partin A, et al. Campbell-Walsh Urology (eleventh Ed.). Elsevier, 2016:692-700.

2. Althof SE, McMahon CG, Waldinger MD, et al. An update of the International Society of Sexual Medicine's guidelines for the diagnosis and treatment of premature ejaculation (PE). J Sex Med 2014;11:1392-422.

3. Serefoglu EC, McMahon CG, Waldinger MD, et al. An evidence-based unified definition of lifelong and acquired premature ejaculation: report of the second International Society for Sexual Medicine Ad Hoc Committee for the Definition of Premature Ejaculation. J Sex Med 2014;11:1423-41.

4. American Psychiatric Association. Diagnostic and Statistical Manual of Mental Disorders. 5th ed. Washington, DC2013.

5. Shindel AW, Althof SE, Carrier S, et al. Disorders of ejaculation: An AUA/SMSNA guideline 2020. 
6. Guo L, Liu Y, Wang X, et al. Significance of penile hypersensitivity in premature ejaculation. Sci Rep 2017;7:10441.

7. Kim JJ, Kwak TI, Jeon BG, et al. Effects of glans penis augmentation using hyaluronic acid gel for premature ejaculation. Int J Impot Res 2004;16:547-51.

8. Salonia A, Bettocchi C, Carvalho J, et al. EAU guidelines on Sexual and Reproductive Health. Presented at the 35th EAU Annual Congress Amsterdam.: EAU guidelines Office; 2020.

9. Kwak TI, Jin MH, Kim JJ, et al. Long-term effects of glans penis augmentation using injectable hyaluronic acid gel for premature ejaculation. Int J Impot Res 2008;20:425-8.

10. Littara A, Palmieri B, Rottigni V, et al. A clinical study to assess the effectiveness of a hyaluronic acid-based procedure for treatment of premature ejaculation. Int J Impot Res 2013;25:117-20.

11. Abdallah H, Abdelnasser T, Hosny H, et al. Treatment of premature ejaculation by glans penis augmentation using hyaluronic acid gel: a pilot study. Andrologia 2012;44

Cite this article as: Kosseifi F, Chebbi A, Raad N, Ndayra A, El Samad R, Achkar K, Durand X, Noujeim A. Glans penis augmentation using hyaluronic acid for the treatment of premature ejaculation: a narrative review. Transl Androl Urol 2020;9(6):2814-2820. doi: 10.21037/tau-20-1026
Suppl 1:650-3.

12. Alahwany A, Ragab MW, Zaghloul A, et al. Hyaluronic acid injection in glans penis for treatment of premature ejaculation: a randomized controlled cross-over study. Int J Impot Res 2019;31:348-55.

13. Gao J, Xu C, Zhang J, et al. Effects of adult male circumcision on premature ejaculation: results from a prospective study in China. Biomed Res Int 2015;2015:417846.

14. Gallo L. The prevalence of an excessive prepuce and the effects of distal circumcision on premature ejaculation. Arab J Urol 2017;15:140-7.

15. Yang Y, Wang X, Bai Y, et al. Circumcision does not have effect on premature ejaculation: A systematic review and meta-analysis. Andrologia 2018. doi: 10.1111/and.12851.

16. Jeong HG, Ahn ST, Kim JW, et al. Practice patterns among Korean urologists for glans penis augmentation using hyaluronic acid filler in the management of premature ejaculation. Sex Med 2018;6:297-301. 